\title{
Multilevel Physical Optics Algorithm for Fast Solution of Scattering Problems Involving Nonuniform Triangulations ${ }^{\dagger}$
}

\author{
Levent Gürel $^{*(1,2)}$ and Alp Manyas ${ }^{(1)}$ \\ ${ }^{(1)}$ Department of Electrical and Electronics Engineering \\ ${ }^{(2)}$ Computational Electromagnetics Research Center (BiLCEM) \\ Bilkent University, TR-06800, Bilkent, Ankara, Turkey \\ E-mail: \{lgurel, alp\}@ee.bilkent.edu.tr
}

\begin{abstract}
Introduction
Physical optics (PO) technique is a very fast and widely used approximation employed for the computation of electromagnetic scattering from electrically large targets. Nevertheless, further acceleration may be needed in real-life applications, where the computation of the scattering pattern over a range of frequencies and/or angles with a sufficient number of samples is desired. For such applications, a multilevel physical optics algorithm (MLPO) can be used to decrease the computation time [1], [2]. MLPO is based on the evaluation of the PO integral in a multilevel fashion by dividing the surface of a target into triangles and evaluating the PO integral on each triangle. We show that the computational efficiency of the MLPO algorithm can be further increased by employing nonuniform triangulations of the target surface so that the triangle size is not nearly uniform, but instead, is determined by the surface curvature.
\end{abstract}

\section{MLPO Algorithm}

MLPO is based on the fact that PO scattering pattern of any target should be sampled at a rate proportional to the dimensions of that target [1]. When the target surface $S$ is divided into $Q$ non-overlapping smaller subdomains, sampling of each subdomain pattern at a lower sampling rate becomes possible. After computing the subdomain patterns on a coarser grid, each pattern can be interpolated to the finest grid of the whole scatterer. This can be done with a relatively low computational cost if the interpolation is performed locally. Following the interpolation, each subdomain pattern can be aggregated to find the scattering pattern of the entire scatterer. Note that, each subdomain origin will be different than the global origin. Therefore, each subdomain will have a rapid phase oscillation in its scattering pattern. This phase oscillation can be factored out as an $\exp \left(2 j \boldsymbol{k}^{s} \cdot \overline{\boldsymbol{r}}_{q}\right)$ term, where $\boldsymbol{k}^{s}$ is the wavevector of the scattered field and $\bar{r}_{q}$ is the center of the $q^{\text {th }}$ subdomain. This phase term should be removed from each subdomain pattern and should be restored after the interpolation. Let $O\left[\overline{\boldsymbol{r}}_{q}\right]$ denote the multiplication with $\exp \left(-2 j \boldsymbol{k}^{s} \cdot \overline{\boldsymbol{r}}_{q}\right), E\left[\overline{\boldsymbol{r}}_{q}\right]$ denote the multiplication with $\exp \left(2 j \boldsymbol{k}^{s} \cdot \overline{\boldsymbol{r}}_{q}\right)$ and $\mathbf{I}_{\bar{N}_{f} \bar{N}_{\phi}}^{N_{N_{\phi}} N_{\phi}}$ denote the interpolation matrix that increases the number of samples from

\footnotetext{
${ }^{\dagger}$ This work was supported by the Scientific and Technical Research Council of Turkey (TUBITAK) under Research Grant 105E172, by the Turkish Academy of Sciences in the frame-work of the Young Scientist Award Program (LG/TUBA-GEBIP/2002-1-12), and by contracts from ASELSAN and SSM.
} 
$\bar{N}_{f} \times \bar{N}_{\phi}$ points to $N_{f} \times N_{\phi}$ points, where $\bar{N}_{f}$ and $\bar{N}_{\phi}$ are the numbers of frequency and angle samples, respectively, in a coarser grid. Then, the PO operator $\Psi$ that computes the PO scattering from the whole surface $S$ can be written as

$$
\Psi S=\sum_{q=1}^{Q} E\left[\overline{\boldsymbol{r}}_{q}\right] \boldsymbol{I}_{\bar{N}_{f} \bar{N}_{\dot{\phi}}}^{N_{N_{\phi}}} O\left[\overline{\boldsymbol{r}}_{q}\right] \Psi \bar{S}_{q} .
$$

This scheme can also be implemented in a multilevel fashion. For instance, each subdomain in (1) can be decomposed into non-overlapping subdomains recursively until the subdomain size is in the order of $\lambda$. This approach can be written in operator form as

$$
\Psi S=\sum_{q=1}^{Q} E\left[\overline{\boldsymbol{r}}_{q}\right] \boldsymbol{I}_{\bar{N}_{f} \bar{N}_{\phi}}^{N_{f} N_{\phi}} O\left[\overline{\boldsymbol{r}}_{q}\right] \sum_{q=1}^{Q} E\left[\overline{\boldsymbol{r}}_{q}\right] \boldsymbol{I}_{\bar{N}_{f} \bar{N}_{\phi}}^{N_{N_{\phi}} N_{\phi}} O\left[\overline{\boldsymbol{r}}_{q}\right] \cdots \Psi \bar{S}_{q} .
$$

When the subdomain size is in the order of $\lambda$, subdomain patterns can be computed directly with PO integration. The patterns of the remaining upper-level subdomains can be computed by aggregation.

\section{Complexity of the MLPO Algorithm}

Computational complexity of the MLPO algorithm can be found as $C_{1} O\left(R^{2}\right)+C_{2} O\left(R^{2} \log R\right)$ [1]. Since there will be $O\left(R^{2}\right)$ subdomains in the bottom level, computational complexity of evaluating the scattering patterns in this level is $O\left(R^{2}\right)$. The reason is that the required number of samples in the frequency and angle grids is fixed in the bottom level since the size of each subdomain is bounded in terms of $\lambda$. At each aggregation step, local interpolations of scattering patterns from one level to the upper one require $O\left(R^{2}\right)$ operations. As the number of levels will be $O(\log R)$, the computational complexity of all interpolations become $O\left(R^{2} \log R\right)$. Note that the constant $C_{1}$ is usually much greater than $C_{2}$. In other words, the computation of the bottom-level radiation patterns dominates the overall CPU time in MLPO. However, the computational complexity turns out to be $O\left(R^{4}\right)$ for the direct PO integration since there should be $O\left(R^{2}\right)$ integration points and $O(R)$ samples in both frequency and angle.

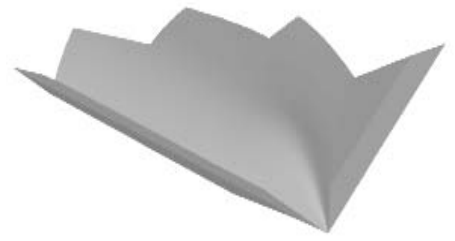

(a)

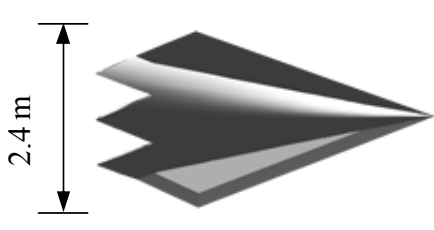

(b)

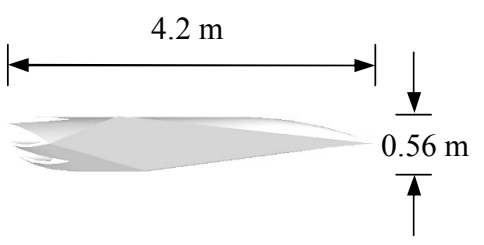

(c)

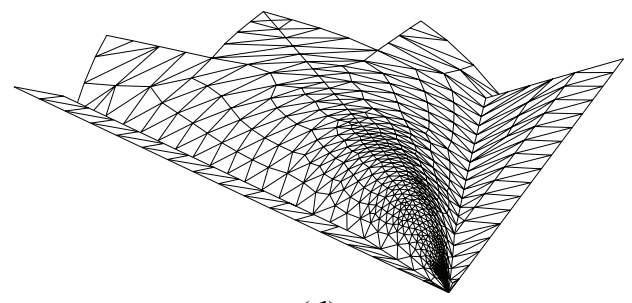

(d)

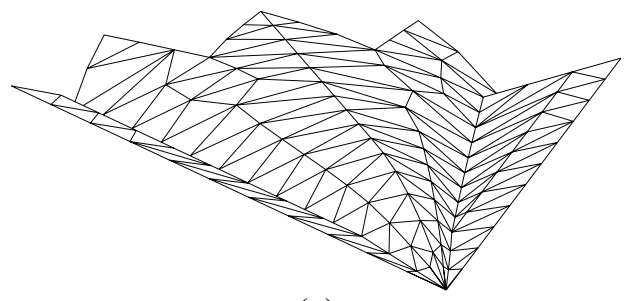

(e)

Figure 1. Geometry of the stealth Flamme target: (a) front, (b) top, and (c) rear view. (d) A nonuniform mesh example. (e) A uniform mesh example. 


\section{Application of MLPO on Nonuniform Triangular Meshes}

We are able to apply the PO method to targets with arbitrarily complicated geometries without sacrificing accuracy. This is because we triangulate the surfaces of complex targets and accurately compute the PO integral on each triangle via an analytical approach [3]. For uniform triangulations, the mesh size is usually chosen to be around $\lambda / 5$ or $\lambda / 10$ depending on the desired accuracy. Alternatively, for nonuniform triangulations, the mesh size is determined by the surface curvature, resulting in fewer triangles. In such a scheme, triangle size gets larger in smooth regions and smaller in curved regions. In Figure 1, both nonuniform and uniform meshes of the airborne stealth target called Flamme [4] are presented as an example.

For a nonuniform mesh, the computation time of the bottom-level radiation patterns decreases as the number of triangles decreases. Hence, the overall computation time of the MLPO also decreases. Note that the subdomains at the bottom level are bounded with $\lambda$ and their scattering patterns are the only scattering patterns that are directly computed with PO integration. Although this is not a problem for a uniform mesh of triangle size $\lambda / 5$ or $\lambda / 10$, there may be "larger" triangles that are too large to fit in bottom-level subdomains in a nonuniform mesh. Since the radiation patterns of those "larger" triangles will have higher oscillations in accordance with their sizes, aggregating them in the bottom level will cause an interpolation error that will grow at each aggregation step. In contrast, computing the scattering patterns of such triangles directly at the finest grid will reduce the computational efficiency. Therefore, radiation pattern of each "larger" triangle should be sampled at a rate proportional to its size and aggregated at the appropriate level, as illustrated in Figure 2.

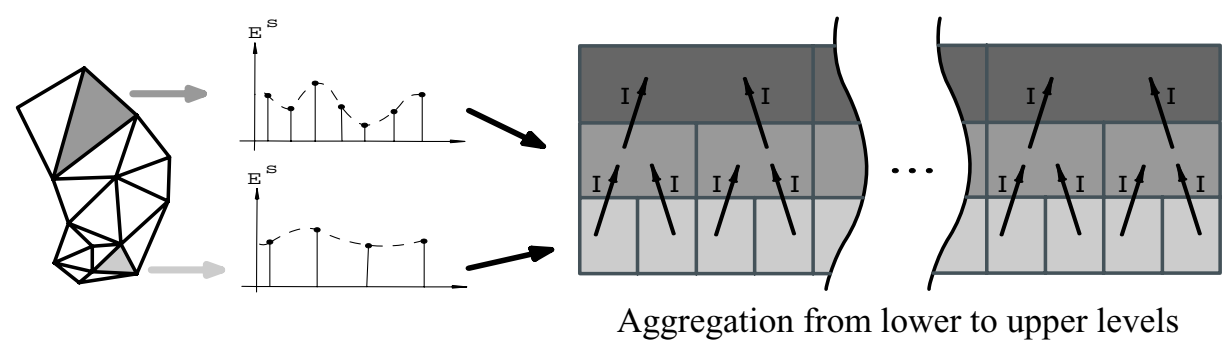

Figure 2. Application of MLPO on a nonuniform triangulation. Patterns of larger triangles are sampled denser and aggregated in upper levels.

To demonstrate the efficiency of MLPO applied on a nonuniform mesh, Flamme geometry given in Figure 1 is illuminated from the back (tail) and backscattering radar cross section (RCS) is computed in the frequency range of 0.1-20 GHz. The uniform mesh of this model, for which the triangle size is approximately $\lambda / 10$ at $5 \mathrm{GHz}$, consists of more than 1,500,000 triangles. In contrast, the nonuniform mesh consists of approximately 175,000 triangles. The CPU time of the PO integration with the uniform mesh is $73 \mathrm{~min}$, whereas the CPU time of the MLPO with the same mesh is only $2.1 \mathrm{~min}$. Furthermore, the CPU time of MLPO with the nonuniform mesh is $0.43 \mathrm{~min}$. Figure 3 shows that, up to $5 \mathrm{GHz}$, PO, MLPO, and nonuniform MLPO results are in good agreement. Above $5 \mathrm{GHz}$, the uniform mesh becomes insufficient. Therefore, the nonuniform mesh is expected to provide more accurate results since it contains smaller triangles in the curved regions of the target surface. 

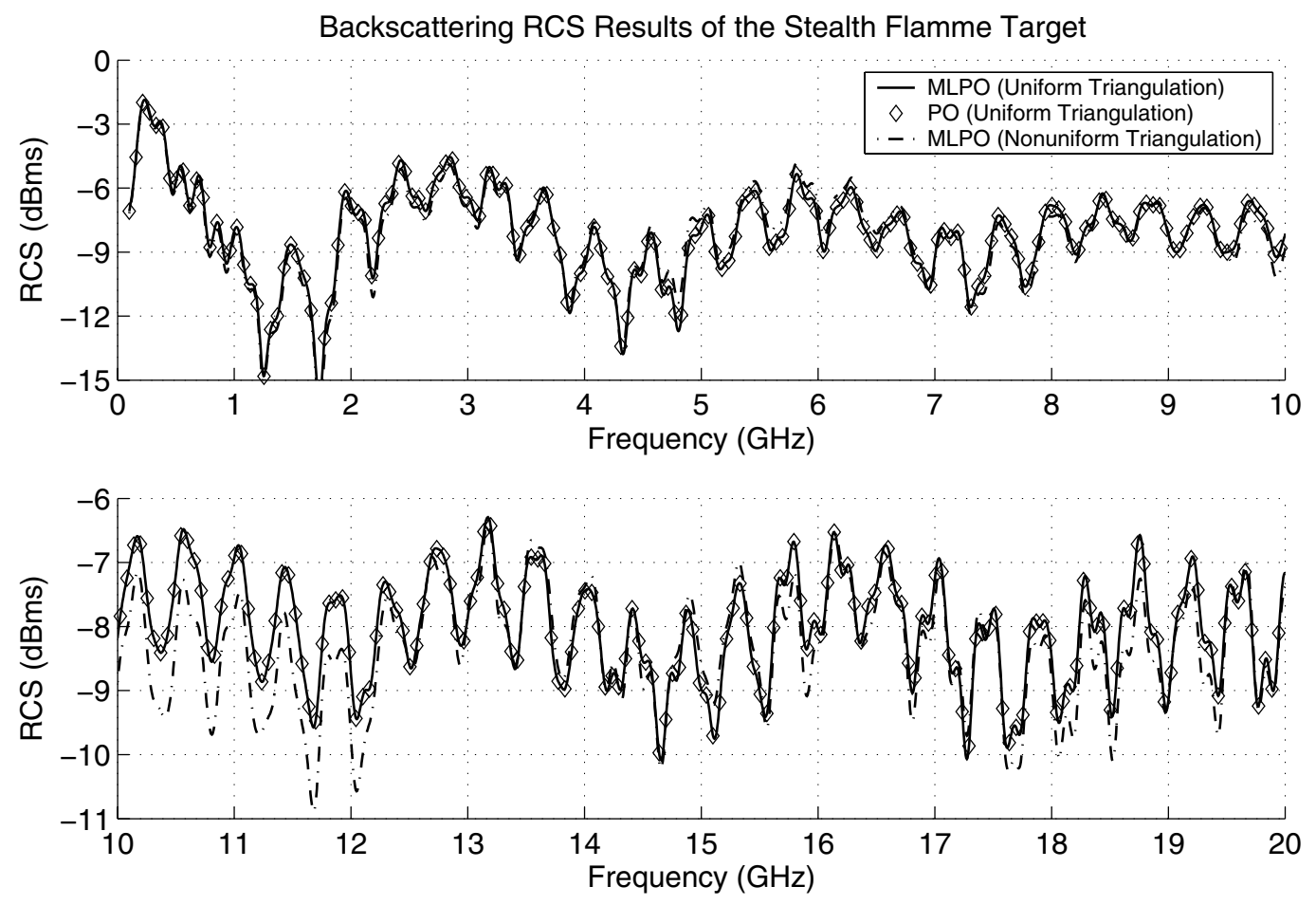

Figure 3. Backscattering RCS results of the stealth Flamme geometry shown in Figure 1.

\section{Conclusion}

The use of MLPO on uniform and nonuniform triangulations of a stealth target is demonstrated for RCS computations. It is shown that, a speedup of approximately 35 can be achieved when MLPO is employed. It is also shown that an additional speedup of approximately 5 can be achieved when nonuniform mesh is used in MLPO instead of a uniform mesh. It should be noted that a uniform mesh of size $\lambda / 10$ at the highest frequency of interest $(20 \mathrm{GHz})$ would contain 24,000,000 triangles. Electromagnetic computations aside, even the generation of so many triangles with today's computer resources is a cumbersome task. Furthermore, the PO computations are performed accurately since the PO integrals on triangles are evaluated analytically.

\section{References}

[1] A. Boag, "A fast physical optics (FPO) algorithm for high frequency scattering," IEEE Trans. Antennas Propagat., vol. 52, pp. 197-204, Jan. 2004.

[2] A. Boag and E. Michielssen, "A fast physical optics (FPO) algorithm for doublebounce scattering," IEEE Trans. Antennas Propagat., vol. 52, pp. 205-212, Jan 2004.

[3] W. B. Gordon, "Far-field approximations to the Kirchhoff-Helmholtz representation of scattered fields," IEEE Trans. Antennas Propagat., vol. 23, pp. 590-592, July 1975.

[4] L. Gürel, H. Bağc1, J. C. Castelli, A. Cheraly, and F. Tardivel, "Validation through comparison: measurement and calculation of the bistatic radar cross section (BRCS) of a stealth target," Radio Science, vol. 38, no. 3, pp. 12-1-12-10, June 2003. 